\title{
An epidemiological application of Popper's method
}

\author{
DOUGLAS L WEED
}

From the National Cancer Institute, 9000 Rockville Pike, Blair Building-Room 301 D, Bethesda, MD 20205, USA

SUMMARY The strong logicodeductive method of science is applied in an investigation of the mortality of a large population of communications workers (338 306 white men) to explain the mortality deficits so often observed in occupational studies. A theory for the healthy worker effect is proposed, and its predictions are tested in comparisons of mortality curves fitted to Weibull functions using regression techniques. The theory, based on the effect of selection on prevalent chronic disease, successfully predicts that as age increases the mortality rates of an employed population soon after hire will diverge from those of a general population. This new finding was observed for mortality from all causes, all causes minus accidents, cardiovascular diseases, and cancers. The theory successfully predicts the rate of decrease of the healthy worker effect as duration of service increases and the relative magnitudes of the effect by cause. Having survived initial empirical testing, this explanation for the effects of selection on occupational mortality meets at least two criteria (only one being sufficient) for deciding whether an explanation is to be preferred: it has made more precise predictions, and it has made new predictions not previously suggested.

Ten years ago, Buck introduced the philosophy of Karl Popper to epidemiology. ${ }^{1}$ In that seminal effort, Popper's views on logic and scientific method $^{2-4}$ were summarised and an example of the use of the hypotheticodeductive method was presented. The example included predictions deduced from a general hypothesis that proposed an infectious origin for cervical cancer; results of a variety of epidemiological investigations provided tests of these predictions.

This paper will continue along the path that Buck has forged and apply this same method, also known as Popper's method of conjectures and refutations, to a different problem in epidemiology, the problem of selection and its effect on occupational mortality. Epidemiologists have long been aware of the so-called "healthy worker effect" (HWE), a phenomenon in which the mortality of an employed population is reduced relative to that of a general population. ${ }^{5}$ The HWE is thought to arise as a result of selection processes; in other words, selection processes may yield an employed population healthier than the general population from which it was selected. This description of the HWE makes intuitive sense. Firstly, a certain proportion of the general population is chronically ill or disabled and therefore is unemployable. Secondly, workers who become ill to the degree that further employment is impossible must leave the workforce. Accordingly, two selection processes are recognised: primary selection refers to that selection which occurs at the time of hire whereas secondary selection refers to that selection which occurs during employment. ${ }^{6}$ Although many additional factors may be responsible for certain characteristics of the HWE, this paper will focus on primary and secondary selection and the factors of age and duration of employment; specifically, it will critically examine the relation between these and all-causes mortality as well as the mortality due to all-causes mortality minus accidents, cancers, and cardiovascular diseases.

\section{Hypotheses and predictions for the healthy worker effect}

Central to Popper's methodology is the careful $a$ priori description of the general hypothesis and predictions to be tested.

As mentioned earlier, there are two types of selection in the workforce: primary and secondary. As a point of departure, consider the effects of entry (ie, primary) selection alone. The following are assumed:

1 A large, age stratified year-of-hire cohort.

$2100 \%$ follow-up on vital status.

3 No hazardous exposures in the workplace.

4 Selection acts to exclude, at the least, prevalent chronic disease. 
These four assumptions lead to the following predictions:

A For comparisons made between the mortality of the cohort soon after hire and the mortality of a general population (eg, the US population of comparable ages, race, and sex):

1 The cohort mortality will be less than the general mortality at all ages.

2 The difference between the mortality rates will increase with increasing age if in the general population the prevalence of disease increases with increasing age.

3 For those diseases with a considerable pre-diagnosis symptomatology (like most cardiovascular disease), deficits in mortality will be greater than those for diseases without such symptomatology (like most cancers).

B For comparisons between the mortality of the cohort and the mortality of the general population made over time, ie, as duration since hire increases:

1 The cohort mortality will approach that of the general population, although it may never reach that level, due to the presence of the aforementioned pool of unemployables.

The actual observations to be made in this investigation will not be on a large year-of-hire cohort. Rather, they will be made on a cross-section of a population, ie, a population comprised of a variety of fragments of past year-of-hire cohorts which has been "exposed" to both primary and secondary selection processes. Accordingly, the assumptions will be expanded, especially regarding the relation of the magnitude and extent of primary selection to that of secondary selection. The assumptions are these:

1 A large, age stratified employed population.

2 Observations are made over a short period of time: a single year.

3 There is incomplete follow up: ie, if a worker leaves active employment and is not vested, then that worker is lost to follow-up.

4 No hazardous exposures in the workplace.

5 Selection, whether primary or secondary, acts to exclude, at the least, prevalent chronic disease.

6 Magnitude of primary selection is a constant over time.

7 Primary selection determines the maximum deficit in mortality.

8 For any period of observation, primary selection is greater than secondary selection.

9 Secondary selection diminishes as time since hire increases.

10 There is a mathematical relation between mortality rates and age.
This final assumption requires elaboration, in part because the notion that age and mortality are mathematically related has been of interest for at least the past 150 years since Gompertz published his law of mortality in 1825 . He related mortality rates, $m$, to age, $x$, in this exponential form: $m=a^{*} \exp (b x)$, where $a$ and $b$ are constants. Gompertz also noted the possible addition of a constant term, $c$, to his model such that it becomes $m=a^{*} \exp (b x)+c$, a feature published by Makeham in the $1860 \mathrm{~s}^{8} \mathrm{~A}$ more recent model, the Weibull curve, expresses mortality as a power function of age: $m=a x^{b}$. These models share a long tradition as curve forms for mortality in human populations and were expected to be particularly effective for the age range of interest.

There is a remarkable similarity between the Gompertz and Weibull models. For example, the basic exponential component of the Gompertz model, $e^{x}$, can also be expressed as an infinite sum of the basic power component of the Weibull model, $x^{b}$, in this Taylor Series expansion: ${ }^{17} e^{x}=\sum_{b=0} x^{b}$. In this investigation, the Weibull model of mortality plus optional Makeham constants was assumed.

The following predictions ensue (these are presented in mathematical form in Appendix I):

A For comparisons made between the mortality of $\frac{}{\Phi}$ the most recently hired subgroup of the employed population and the mortality of a general population:

1 The mortality of this subgroup will be less than that of the general population at all ages (Prediction A1).

2 The difference in mortality between these two populations will increase with increasing age (Prediction A2).

3 For those diseases with a considerable pre-diagnosis symptomatology (like most cardiovascular diseases), deficits in mortality will be greater than those for diseases without such symptomatology (like most cancers) (Prediction A3).

Note that the first three predictions are identical with those made for a year-of-hire cohort soon after hire.

B For comparisons made between the various fragments of year-of-hire cohorts, ie, for comparisons made between subgroups of the employed population stratified by duration of employment:

1 There will be observed a gradually diminishing rise in mortality across successive subgroups. That is, if there are three such subgroups, one with $x$ years of service, one with $x+n$ years of service, and one with $x+2 n$ years of service, then the mortality of a subgroup with $x$ years of 
service will be less than that of a subgroup with $x+n$ years of service and the difference in mortality between these two subgroups will be greater than that observed between the subgroup with $x+n$ years of service and that with $x+2 n$ years of service (Prediction B1).

2 This rise in mortality will be less rapid overall for diseases with a considerable pre-diagnosis symptomatology than for diseases without such symptomatology (Prediction B2).

In summary, the predictions are: there is a persistent effect of selection upon age-specific mortality in that the HWE will be observed at all ages. Further, this age effect soon after primary selection, by virtue of the fact that considerably more prevalent chronic disease exists at older ages, is one that will show an increasing difference in mortality across age. Also there is a duration of employment effect in that there will be observed a diminishing rise of employed population mortality toward the general mortality. Finally, there is a cause of death effect in which cardiovascular disease mortality will show greater deficits and slower rises than will cancer mortality. Note that these predictions do not consider the relation of the employed population's mortality to the general population's mortality as duration of service increases: for example, no prediction is made regarding the appearance of an increasing difference in mortality (general minus employed) for subgroups with longer durations of employment.

\section{Empirical testing: materials and procedures}

Two problems have plagued many of the reports ${ }^{1011}$ of the HWE: firstly, the relatively small sizes of the study populations have not provided stable age-specific mortality rates when stratified by duration of employment and by cause of death. Secondly, the study populations are often exposed to physical or chemical hazards, the effects of which are mixed with those of selection. Both of these problems, which in part are responsible for the recently expressed call for more studies of the HWE, ${ }^{12-14}$ could be overcome by investigating the HWE in a large, unexposed working population. For this investigation, the employees of a large communications firm in the US were felt adequately to represent just such a population.

The study population, 338306 actively working white male employees, is a subset $(38.4 \%)$ of the population included on a database derived both from encoded demographic and work-history files and from a mortality data file on employees of the affiliated operating telephone companies of the Bell System as organised before 1 January 1984.
Demographic information (eg, date of birth, race, and sex) and work-history information (eg, dates of service) were obtained from company personnel files. The mortality data file, developed at the American Telephone and Telegraph Company, maintains death certificate information for active employees and pensioners who died within the period 1 January 1976 to 31 December 1978 . Causes of death were nosologist-coded using the Eighth International Classification of Disease. A single year of follow-up (1976) was examined in this initial study; additional studies over a full three years are expected. The database, formed by computer links between demographic, work-history, and mortality files, does not maintain information on individuals who die after leaving the firm unless they are eligible for the death benefits program, nor for part-time, temporary, occasional or New York City employees.

Thus, the study population represents those active white male workers, aged 20 to 64 , who worked in a full-time position sometime during the year 1976 . The age distribution of this population and the observed deaths are shown in table 1 . Mortality rates of an incidence-density form ${ }^{15}$ were calculated for the year 1976 by five year age groups (range 20-64 years) and 15 year duration of service groups (range 0-44 years) cross-classified by the cause of death categories given in Appendix I.

Two curve-fitting techniques were used: weighted linear and weighted nonlinear least squares regression. Inverse variance weights were used; the variance is given by the deaths divided by the square of the person-years. ${ }^{16}$ While descriptions of these curve-fitting techniques are available in theory ${ }^{17} 18$ and in computer software applications, ${ }^{19}$ some basic notions are presented here nevertheless. Linear least squares regression requires that the underlying functional form to be fit to the data is linear, or at the least is transformable into a linear equation of the form: $y=m x+b$; where $m$ is the slope and $b$ is the

Table 1 Age distribution of person-years and observed deaths*

\begin{tabular}{llrrrr}
\hline \multicolumn{5}{c}{ Deaths } \\
\cline { 3 - 6 } Age $(y r)$ & Person-years & ALL & AMA & CAN & CVD \\
\hline $20-24$ & 26817 & 24 & 11 & 5 & 0 \\
$25-29$ & 63945 & 43 & 23 & 9 & 3 \\
$30-34$ & 52372 & 44 & 28 & 10 & 9 \\
$35-39$ & 33922 & 32 & 23 & 8 & 9 \\
$40-44$ & 40819 & 71 & 64 & 15 & 35 \\
$45-49$ & 44787 & 155 & 151 & 43 & 78 \\
$50-54$ & 40361 & 225 & 208 & 66 & 113 \\
$55-59$ & 21155 & 200 & 193 & 68 & 89 \\
$60-64$ & 5480 & 75 & 72 & 31 & 36 \\
\hline
\end{tabular}

*White male actively working communications workers, 1976.

$A L L=$ all causes; $A M A=a l l$ causes minus accidents; $C A N=$ cancers; CVD $=$ cardiovascular diseases. 
$y$-intercept. Such a transformation is possible for the Weibull model but not for its Makeham counterpart. Once the transformation is accomplished, the values for the parameters which satisfy the criteria for minimisation of the error sum of squares are obtained in a unique (closed) form. In contrast, for nonlinear least squares regression, no such general closed form solution exists; instead, an iterative procedure is employed in which the initial estimates for the parameters are continually changed until the error sum of squares is minimised and the convergence criteria to the minimum have been met.

In this analysis, initial parameter estimates for nonlinear regression were made with the linear regression technique. Weibull-Makeham curves were fit for the 1976 white male US mortality rates and for the study population categorised by the three duration-of-service subgroups.

\section{Results}

Figures 1, 2, 3, and 4 show the results of fitting age mortality curves to the data and will provide the empirical information to which the predictions will be compared. Correlation coefficients after linear regression are shown in table 2 . These improved after nonlinear regression; the value of the coefficient for each curve exceeded 0.96 . For this reason, and to facilitate their visual inspection, only fitted values appear in the figures.

Prediction A1 states that the HWE will be present at all ages in the group with the least amount of accrued service. Failure of this prediction may be inferred in the instance in which the difference of mortality rates is less than or equal to zero. This prediction is upheld for all causes, all causes minus accidents, and for cardiovascular disease mortality yet fails for cancer mortality at the youngest ages. The greatest difference, at ages 20-24 years, is -9.95 $(p<0.05)$, corresponding to a rate ratio of 2.61 .

Prediction A2 asserts that in this same subgroup of shorter durations of service, the HWE increases in absolute magnitude as age increases. Failure of the prediction may be inferred in instances in which the first derivative of the difference function

Table 2 (Correlation coefficients) ${ }^{2}$ by cause and duration of service after linear regression

\begin{tabular}{llll}
\hline & \multicolumn{3}{l}{ Duration of service (years) } \\
\cline { 2 - 4 } Cause of death & Up to 14 & $15-29$ & $30-44$ \\
\hline All causes & 0.37 & 0.99 & 0.98 \\
AMA $^{*}$ & 0.69 & 0.93 & 0.98 \\
Cancers & 0.38 & 0.97 & 0.44 \\
Cardiovascular diseases & 0.97 & 0.98 & 0.98 \\
\hline
\end{tabular}

*All causes minus accidents.
$(M(x)-m(x))$, derived from the nonlinear curves, is less than or equal to zero. Calculated values of these derived functions are presented in table 3 . In each of the four cause categories the prediction is not refuted.

Prediction $A 3$ asserts that the HWE, when measured with the mortality rate difference, will be greater for cardiovascular disease mortality than for cancer mortality in the group with the least amount of accrued service. Table 4 provides this information. Again, at all ages, the prediction is upheld.

Prediction $B 1$ states that a diminishing rise in mortality curves will be observed as duration of service increases, a finding readily confirmed for all causes and all causes minus accidents mortality by visual inspection of figs 1 and 2 respectively.

Table 3 Derivatives of mortality difference functions ${ }^{*}$ by age and cause

\begin{tabular}{|c|c|c|c|c|c|}
\hline Age (yr) & All causes & $A M A$ & Cancers & $C V D$ & 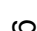 \\
\hline $20-24$ & $23 \cdot 01$ & $22 \cdot 10$ & $6 \cdot 37$ & $4 \cdot 71$ & \\
\hline $25-29$ & $62 \cdot 22$ & $57 \cdot 39$ & $17 \cdot 28$ & $17 \cdot 30$ & \\
\hline $30-34$ & $139 \cdot 43$ & 126.03 & 38.94 & 46.95 & \\
\hline $35-39$ & $271 \cdot 67$ & $245 \cdot 84$ & $76 \cdot 57$ & $106 \cdot 47$ & \\
\hline $40-44$ & $473 \cdot 12$ & 438.97 & $135 \cdot 25$ & $213 \cdot 70$ & \\
\hline $45-49$ & $747 \cdot 42$ & $731 \cdot 90$ & $218 \cdot 32$ & $392 \cdot 35$ & \\
\hline $50-54$ & $1076 \cdot 22$ & $1155 \cdot 34$ & $325 \cdot 01$ & $672 \cdot 72$ & \\
\hline 55-59 & 1403.07 & 1744.03 & $447 \cdot 14$ & $1092 \cdot 52$ & \\
\hline $60-64$ & $1611 \cdot 82$ & 2536.42 & 564.68 & 1697.60 & \\
\hline
\end{tabular}

*Difference function is the fitted curve (US) minus the fitted curve for the communications workers subgroup with up to 14 years of service.

AMA = all causes minus accidents; $C V D=$ cardiovascular diseases.

Table 4 Mortality rate differences* for cancer and $\overrightarrow{\vec{t}}$ cardiovascular diseases

\begin{tabular}{lcc}
\hline Age $(y r)$ & Cancers & Cardiovascular diseases \\
\hline $20-24$ & -9.95 & 0.58 \\
$25-29$ & $-7 \cdot 17$ & 3.30 \\
$30-34$ & -0.42 & 11.67 \\
$35-39$ & 13.63 & $32 \cdot 29$ \\
$40-44$ & $39 \cdot 62$ & $76 \cdot 21$ \\
$45-49$ & $83 \cdot 28$ & 160.67 \\
$50-54$ & $150 \cdot 72$ & 310.95 \\
$55-59$ & 247.03 & 562.59 \\
$60-64$ & 373.83 & 963.80
\end{tabular}

*US rate minus rate of communications workers subgroup with up to 14 years of service after nonlinear regression fits.

Table 5 Comparisons of integral areas between duration of service curves by cause

\begin{tabular}{|c|c|c|}
\hline Cause of death & Area $\left(M_{i+1}-M_{i}\right)$ & Area $\left(M_{i+2}-M_{i+1}\right)$ \\
\hline $\begin{array}{l}\text { All causes } \\
\text { AMA } \\
\text { Cancers } \\
\text { Cardiovascular disease }\end{array}$ & $\begin{array}{r}192 \cdot 12 \\
192 \cdot 46 \\
69 \cdot 94 \\
119 \cdot 42\end{array}$ & $\begin{array}{l}71 \cdot 82 \\
80 \cdot 21 \\
69 \cdot 25 \\
31 \cdot 47\end{array}$ \\
\hline
\end{tabular}

AMA $=$ all causes minus accidents. 
Calculations of the appropriate integral comparisons between curves (ie, across ages 45-64) are presented in table 5. The prediction is confirmed for all four cause categories. Note that in the case of cardiovascular disease mortality (fig 4) there appears

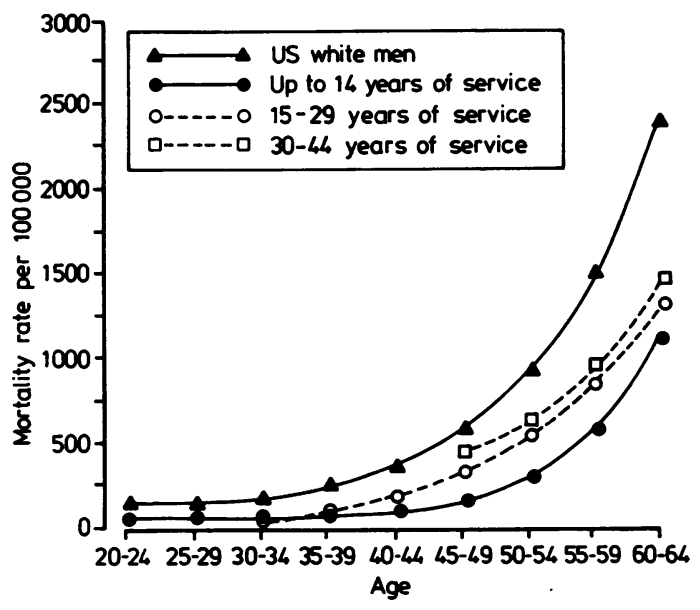

Fig 1 Nonlinear regression fits of all causes mortality curves by age for US white males and for three duration of service subgroups of study population.

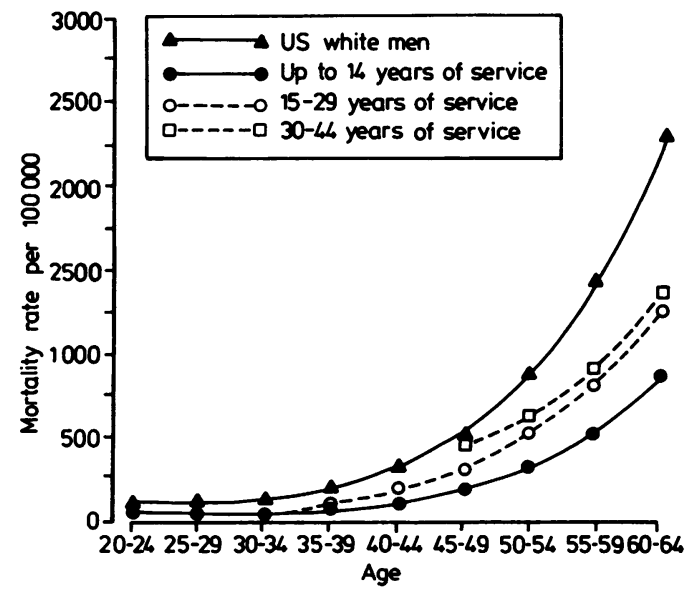

Fig 2 Nonlinear regression fits of all causes mortality minus accidental deaths curves by age for US white males and for three duration of service subgroups of study population. to be a particularly strong selection effect in the oldest ages of the subgroup with 30 to 44 years of service. This is illustrated by the curve dropping below that for the subgroup with 15 to 29 years of service.

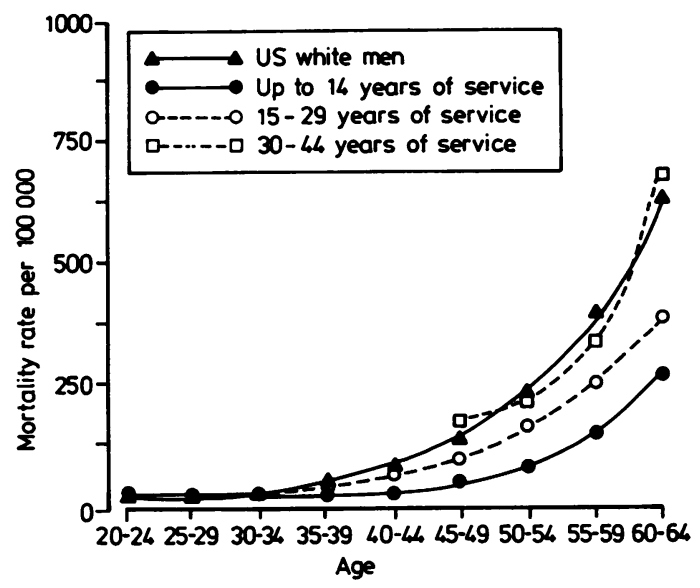

Fig 3 Nonlinear regression fits of cancer mortality curves by age for US white males and for three duration of service subgroups of study population.

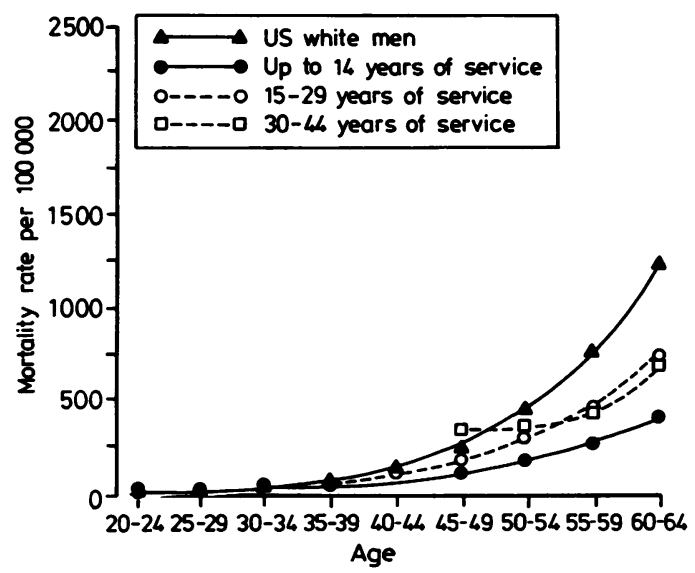

Fig 4 Nonlinear regression fits of cardiovascular disease mortality curves by age for US white males and for three duration of service subgroups of study population. 
Finally, Prediction B2 states that cancer mortality will rise as duration of service increases at a faster rate than will cardiovascular disease mortality. This prediction is refuted if the ratio of the two integral areas defined and enclosed by the three cardiovascular disease duration of service curves is greater than the ratio of the analogous ratio from the cancer curves. In this instance, the cardiovascular disease integral ratio is 0.26 while the cancer integral ratio is 0.99 . Therefore, the rate at which the cancer curves are rising as duration of service increases clearly exceeds the rate of rise of the cardiovascular disease curves; the prediction is not refuted.

\section{Discussion}

As Popper has often noted, science does not begin with theories, rather it begins with problems. ${ }^{20}$ Like any science, epidemiology has its own share of problems, not the least of which is the discovery of the determinants of disease. To many epidemiologists, this is identical with the problem of discovering the causal links between exposure and disease. For occupational epidemiologists, a related problem arises in the form of the healthy worker effect. It is an important problem because the effects of selection interfere with the search for exposure-disease causal chains.

In order better to understand how the problem of the healthy worker effect was approached from a Popperian methodology let us first look at how a more typical epidemiological study of this problem might take place. There would be an initial review of the existing literature on the subject, from which it could be concluded that an investigation comparing the mortality of a large, unexposed working population to the general population would provide relatively precise estimates of the magnitude of the effect unconfounded by the effects of work related exposures. These comparisons could be made and the inherent difficulties of the often used indirect method of mortality rate standardisation (also called the standardised mortality ratio or SMR) could be noted. ${ }^{21}{ }^{22}$ From the results it would be possible to make some statements regarding the characteristics of the healthy worker effect. Many studies have reported such general features; these are summarised as follows: ${ }^{8}$

1 the healthy worker effect is greatest at youngest ages and decreases with increasing age;

2 the healthy worker effect diminishes as duration of service increases; and

3 the healthy worker effect is greater for cardiovascular disease mortality than it is for cancer mortality.
There are others, which relate to other factors such as race and sex, but they do not concern us at this time. What does concern us is the origin of these statements and their relation to a critical scientific methodology.

These characteristics of the healthy worker effect have arisen from observations made on a variety of occupational populations. Therefore, they have arisen inductively; they represent generalisations resulting from an accumulation of similar observations. They could become our predictions for the next study of the healthy worker effect but only if we are justified in making this purely inductive leap from observation to prediction. But as Popper's views suggest, there are serious questions about the validity of this approach. Alternatively, an a priori explanation for the healthy worker effect could be proposed. This explanation would entail a series of assumptions, initial conditions, and deduced predictions about the presumed action of selection on disease and mortality. It may be possible that some of the predictions so derived will be similar to those tha have arisen from observation alone. It is also possible that this a priori explanation will yield some new unexpected predictions. In any case, it is only within critical deductive method, such as that advocated by Popper, that the logical implications of observations are strongly realised; for it is only in such a deductive methodology that the observations actually test the general assertions. Thus, it is also possible that suct an a priori explanation of the healthy worker effec will not hold up under a critical inquiry.

In summary, the first step in nearly every epidemiological study is to review the existing studies and commentaries on the subject. Yet for the healthy worker effect, the conclusions one may draw from such a review are actually an endpoint of an inductive process comprised of a long series of observations with little or no explicit theoretical basis. While a critic may insist that there is in fact a theoretical basis for the healthy worker effect (that it is the result of selection processes), the essential link between this general theoretical statement and the predictions that can be derived from it remains unstated.

Any explanation which arises in an attempt to fill this gap must withstand the rigours of the critical deductive method; its general assumptions and initial conditions must first be explicated, and the predictions must then be derived from them. These predictions represent the theoretical expectations for that which is to be observed, and it follows from the basic principles of deductive logic that they can (and should) be rejected if they fail to match the observations. Such rejections, also called refutations, are vital to scientific progress because they pave the way for new explanations, explanations which also 
will be tested. It is important to evaluate this quasi-evolutionary progress by examining how each explanation (relative to either its predecessors or current competitors) meets at least one of the following criteria: ${ }^{13}$

(i) It makes more precise predictions.

(ii) It explains more of the previous observations, and in more detail.

(iii) It has passed tests which others have failed.

(iv) It has suggested new tests.

(v) It has unified or connected phenomena not previously considered to be related.

For the explanation of the healthy worker effect proposed here, the concordance of the predictions and the observations is shown in table 6. Overall, the observations correspond extremely well with the predictions made for them. Thus it has been shown here that a logically consistent and testable explanation for the healthy worker effect has successfully predicted the mortality patterns observed in an epidemiological investigation of actively working communications workers. It has identified the separate and different effects of selection on mortality by age and by duration of employment, effects that were phrased in terms of measures not commonly used in occupational mortality studies. Specifically, it used rate differences to measure trends and derived and integrated functions to test epidemiological predictions. Therefore, it appears that at least two of the evaluative criteria mentioned above were satisfied. This explanation has made more precise predictions, and by suggesting new tests it has made new predictions not previously suggested.

Recall that because these are relativistic criteria we need to defend this claim by comparing the explanation posed in this paper with at least one of its predecessors. Consider, for example, a study of the healthy worker effect in workers exposed to vinyl chloride. $^{10}$ There it was suggested that three

Table 6 Concordance of observations with predictions

\begin{tabular}{lll}
\hline Prediction & Cause category & Agrees? \\
\hline A1 & ALL & + \\
& AMA & + \\
& CAN & + \\
A2 & CVD & + \\
& ALL & + \\
& AMA & + \\
A3 & CAN & + \\
B1 & CVD & + \\
& CAN \& CVD & + \\
& ALL & + \\
B2 & AMA & + \\
& CAN & + \\
& CVD & + \\
\end{tabular}

$\mathrm{ALL}=$ all causes; $\mathrm{AMA}=$ all causes minus accidents; $\mathrm{CAN}=$ cancers; CVD = cardiovascular diseases. properties of the healthy worker effect are important to measure: selection, survival, and length of follow-up. While these effects were carefully isolated and measured in this study, little, if any, attempt was made to predict characteristics of these effects $a$ priori. Although it was suggested that an explanation for the healthy worker effect should incorporate Ogle's three properties, no such explanation was put forward. Thus, in contrast, the explanation posed herein provides a scientific framework for the effects of selection on occupational mortality. It incorporates both static selection (Ogle's selection effect) and dynamic selection (Ogle's survival effect). It also incorporates age, duration of service, and cause-of-death effects. However, most important, it provides $a$ priori testable predictions for these effects based on simple properties of selection. And it suggests new measurement techniques. It is on this basis that it can be asserted that this explanation satisfies the Popperian criteria of more precise predictions and new tests.

Nevertheless, this explanation for the healthy worker effect is not complete, ie, it was not entirely successful. It failed to predict an excess of cancer mortality at the youngest ages and it failed to detect a strong selection effect for cardiovascular disease mortality in older workers with long service durations. And, because a central notion in the methodological approach advocated by Popper is that the lack of a concordance between prediction and observation must reflect back on the theory from which the prediction arose, it is therefore necessary to question the assumptions that selection simply acts to exclude prevalent chronic disease ${ }^{23}$ and that secondary selection simply diminishes as time since hire increases.

While it is possible to rescue this theory from these failures with appropriate ad hoc alterations, such a 'conventionalist' stratagem threatens to destroy its claim to scientific status. In the end we must conclude that this theory for the healthy worker effect is not final nor ultimate. Nevertheless it has been shown to be an informative representation of reality, given the scope of its assumptions and the intensity of the attempts to refute it. In future critical investigations, it may be rejected in full or some part of it may well be subsumed under some more general theory; the progress of the science of epidemiology is not best characterised by its inflexibility, rather by its potential for change.

The comments and suggestions made by Michael Selmon are gratefully acknowledged; also the support given by Professors H A Tyroler, Carl Shy, Ralph Patrick, Harvey Checkoway, and M J Symons, and the assistance of Patti Achy and Gayle Marwine. 


\section{References}

${ }^{1}$ Buck C. Popper's philosophy for epidemiologists. Int J Epidemiol 1975; 4: 159-68.

${ }^{2}$ Popper KR. The logic of scientific discovery. New York: Harper and Row, 1968.

${ }^{3}$ Popper KR. Science: conjectures and refutation. In: Conjectures and refutations: The growth of scientific knowledge. New York: Harper and Row, 1968; 33-65.

${ }^{4}$ Popper KR. Objective knowledge: an evolutionary approach. Oxford: Clarendon Press, 1981.

${ }^{5}$ McMichael AJ. Standardised mortality ratios and the "healthy worker effect": scratching beneath the surface. J Occup Med 1976; 18: 165-8.

${ }^{6}$ Olsen J. Some methodologic problems encountered in occupational health research. Scand J Soc Med 1981; 9: 19-24.

${ }^{7}$ McMichael AJ, Haynes SG, Tyroler HA. Observations on the evaluation of occupational mortality data. J Occup Med 1975; 17: 128-31.

${ }^{8}$ Smith D, Keyfitz N. Mathematical demography. New York: Springer-Verlag, 1977.

${ }^{9}$ Fisher RC, Ziebur AD. Calculus and analytic geometry. Englewood Cliffs, NJ: Prentice-Hall, 1965; 734.

${ }^{10}$ Fox AJ, Collier P. Low mortality rates in industrial cohort studies due to selection for work and survival in the industry. Br J Prev Soc Med 1976; 30: 225-30.

${ }^{11}$ McMichael AJ, Spirtas R, Kupper LL. An epidemiologic study of mortality with a cohort of rubber workers 1964-1972. J Occup Med 1974; 16: 458-64.

${ }^{12}$ Hernberg S. Epidemiology in occupational health. In: Developments in occupational medicine. Chicago: Year Book Medical, 1980; 3-40.

${ }^{13}$ Occupational mortality: The Registrar General's Decennial Supplement for England and Wales, 1970-72. Office of Population Censuses and Surveys. London: HMSO, 1978.

${ }^{14}$ Vinni K, Hakama M. Healthy worker effect in total Finnish population. $B r J$ Ind Med 1980; 37: 180-4.

${ }^{15}$ Morgenstern H, Kleinbaum DG, Kupper LL. Measures of disease incidence used in epidemiologic research. Int J Epidemiol 1980; 9: 97-104.

${ }^{16}$ Yule GU. On some points relating to vital statistics, more especially statistics of occupational mortality. J Roy Stat Soc 1934: XCVII: Part 1, 1934.

${ }^{17}$ Kleinbaum DG, Kupper LL. Applied regression analysis and other multivariable techniques. North Scituate, Massachusetts: Duxbury, 1978.

${ }^{18}$ Bard Y. Nonlinear parameter estimation. New York: Academic Press, 1974.

${ }^{19}$ SAS User's Guide. Cary, North Carolina: SAS Institute Inc., 1979.

${ }^{20}$ Popper KR. Science: problems, aims, responsibilities. Fed Proc 1963; 22: 961-72.

${ }^{21}$ Redmond CK, Breslin PP. Comparison of methods for assessing occupational hazards.J Occup Med 1975; 17: 313-7.

${ }^{22}$ Chiazze L. Problems of study design and implementation of industrial mortality experience. J Occup Med 1976; 18: $169-70$.

${ }^{23}$ Wen CP, Tsai SP, Gibson RL. Anatomy of the healthy worker effect: A critical review.J Occup Med 1983; 25: 283-9.

\section{Appendix I}

A distinction can be made between what is known as a conceptual hypothesis, such as factor $A$ is associated with disease $\mathrm{B}$, and a working hypothesis such as the incidence of disease $B$ in a population exposed to factor $A$ is greater than the incidence of disease $B$ in a population not so exposed. This is an oversimplification, to be sure, but it still serves to point out that a working hypothesis is more easily tested.

The predictions for the effects of selection on cross-sectional mortality can also be converted, but not from conceptual hypotheses to working hypotheses, rather from conceptual predictions to working predictions. These conversions involve a minimum of additional assumptions.

Firstly, some definitions are presented:

$\mathbf{M}=$ mortality rate of the general population

$m=$ mortality rate of the employed population

$i=i$ th 15 -year duration of service stratum $i=1,2$, 3 (0-44 years)

$\mathrm{j}=\mathrm{jth} 5$-year age stratum $\mathrm{j}=1,2, \ldots, 9(20-64$ years)

$x=$ age

ALL $=$ all causes (ICD codes 000-999)

AMA $=$ all causes minus accidents (ICD codes 000-799 and 950-999)

CAN $=$ malignant neoplasms $($ ICD codes 140-209)

$\mathrm{CVD}=$ cardiovascular disease (ICD codes 390-448)

$\mathrm{D}(\mathrm{m}(\mathrm{x}))=$ derivative of function $\mathrm{m}(\mathrm{x})$ relative to $\mathrm{x}$.

$x$ Measures the rate of change of $m(x)$ relative to $x$.

d

$\S m(x) d x=$ integral of the function $m(x)$ evaluated c from $c$ to $d$.

Measures the area under the curve $f(x)$ from point $x=c$ to point $x=d$.

Five major conceptual predictions were asserted; these are restated as working predictions:

\section{Conceptual prediction A1}

For the group with the shortest duration of service, their mortality will be less than the general population mortality, at all ages.

Working prediction $A 1$ (applies to ALL, CAN, CVD, AMA)

Difference: $\quad\left(\mathrm{M}_{1, \mathrm{j}}-\mathrm{m}_{1, \mathrm{j}}\right)>0$ for all $\mathrm{j}$

Ratio: $\quad\left(\mathrm{m}_{1, j} / \mathrm{M}_{1, \mathrm{j}}\right)<1$ for all $\mathrm{j}$

\section{Conceptual prediction $A 2$}

The difference in mortality between the group with the shortest duration of service and the general population will increase with increasing age. 
Working prediction $A 2$ (applies to ALL, CAN, CVD, AMA)

$\mathrm{D}_{\mathrm{x}}\left(\mathrm{M}_{1, \mathrm{j}}(\mathrm{x})-\mathrm{m}_{1, \mathrm{j}}(\mathrm{x})\right)>0$

\section{Conceptual prediction $A 3$}

For the group with the shortest duration of service, CVD mortality deficits will be greater than CAN mortality deficits.

Working prediction A3 (applies only to CAN, CVD) $\left(M_{1, j}-m_{1 . j}\right)_{C V D}>\left(M_{1, j}-m_{1, j}\right)_{\text {cAN }}$ for all $j$

\section{Working hypothesis B1}

For simultaneous comparisons of duration of service groups, a gradually diminishing rise in mortality curves will be observed.
Working prediction $B 1$ (applies to ALL, CAN, CVD, AMA; limits of integration: ages 45-64 (ie, $6 \leqslant j \leqslant 9)$ )

$$
\S m_{i+1, j}(x) d x-\S m_{i, j}(x) d x>\S m_{i+2, j}(x) d x-\S m_{i+1, j}(x) d x
$$

\section{Conceptual prediction B2}

For simultaneous comparisons of duration of service groups, the rise in mortality will be less rapid for cardiovascular diseases than for malignant neoplasms.

Working prediction B2 (applies to CAN, CVD; limits of integration: ages $45-64(\mathrm{ie}, 6 \leqslant j \leqslant 9)$ )

$$
\begin{array}{r}
\quad\left[\S m_{i+2, i}(x) d x-\S m_{i+1, j}(x) d x\right]_{C V D} /\left[m_{i+1, j}(x) d x-\right. \\
\left.\S m_{i, j}(x) d x\right]_{\text {CVD }} \\
<\left[\S m_{i+2, j}(x) d x-\S m_{i+1, j}(x) d x\right]_{\text {CAN }} /\left[m_{i+1}(x) d x-\right. \\
\left.\S m_{i, j}(x) d x\right] \text { CAN }
\end{array}
$$

